\title{
Beneficios de una temporada de Educación Deportiva en las relaciones de género
}

\author{
Benefits of a Sport Education Season on Gender Relations
}

\author{
Lucía Reyes' ${ }^{1}$ \\ Luis Miguel García-López * (i) \\ Rafael Justo Sánchez-Ajofrín ${ }^{10}$

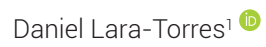

1. Universidad de Castilla-La Mancha, España.

\section{Resumen}

Las relaciones entre iguales son un factor clave en el desarrollo social de los preadolescentes. Por ello el presente trabajo tiene como objetivo el estudio de las relaciones sociales entre ambos géneros. Además, también se estudia la posible repercusión que sobre estas puede tener la implementación de una unidad didáctica que utiliza como metodología el modelo de Educación Deportiva (ED) en una clase de sexto de Educación Primaria. Para la implementación de esta temporada se diseñó un programa compuesto de diez sesiones estructuradas según el modelo de ED y tres actividades complementarias. Del mismo modo, para poder realizar una comparación final de las relaciones entre ambos géneros, se utilizan instrumentos de evaluación en diversos momentos del estudio, antes y después, tales como test sociométricos o entrevistas. Los datos cuantitativos fueron analizados a través de la prueba t de student para muestras relacionadas y los datos cualitativos a través de un análisis de contenido. Los resultados muestran un marcado rechazo inicial entre ambos géneros. Tras la implementación del programa, se refleja una leve mejora en cuanto a la aceptación del género contrario y la disminución de su rechazo, a lo que contribuyen pilares básicos de la ED. Los motivos que los alumnos enuncian para aceptar o rechazar al género contrario son clasificados dentro de seis categorías (comportamiento, amistad, compañerismo, dominancia, falta de competencia y otros). Asimismo, el conocimiento de una clara situación de rechazo inicial y la posterior mejora de las relaciones sociales reflejan los logros conseguidos con el programa implementado a través del modelo de ED. En este sentido, para mejorar estos resultados en futuros estudios, es importante que se realice un trabajo continuado en el tiempo y el modelo sea reforzado con el uso de otras técnicas complementarias que favorezcan el trabajo de las relaciones sociales.
\end{abstract}

Palabras clave: Educación Deportiva, estereotipos de género, equidad, Educación Física.

\begin{abstract}
Peer relationships are a key factor in the social development of tweens thus the present work aims to study the social relations between both genders. In addition, we also studied the possible repercussion of the implementation of a unit which uses as methodology, the sport education pedagogical model with year-6 Primary School students. For the implementation of this unit, a ten session's program structured according to SE and three complementary activities was designed. In the same way, we used sociometric tests and interviews to analyze differences between the initial and the final situation. The data analyzed through a student's t test for related samples and a qualitative analysis, showed a marked rejection between boys and girls. After the implementation of the program, findings reflected a slight improvement in terms of acceptance of the opposite gender and the reduction of its rejection, to which basic pillars of SE, such as affiliation and festivity contributed to. The motives that the students enunciate to accept or reject the opposite sex can be classified into six categories (behavior, friendship, companionship, dominance, lack of competence and others). Likewise, the knowledge of a clear situation of initial rejection and the subsequent improvement of social relationships reflect the achievements made with the program implemented through the SE model. In this sense, to improve these results in future studies, it is important that continuous work is carried out over time and the model be reinforced with the use of other complementary techniques, which foster social relations.
\end{abstract}

Keywords: Sport Education, gender stereotypes, equity, Physical Education.

*Autor de correspondencia: Lucía Reyes, lucia.reyes@uclm.es

Recibido: 08 de octubre de 2020

Aceptado: 11 de diciembre de 2020

Publicado: 31 de enero de 2021

Como citar (APA): Reyes, L., García-López, L.M., Sánchez-Ajofrín, R.J., \& Lara-Torres, D. (2021). Beneficios de una temporada de Educación Deportiva en las relaciones de género. JUMP, (3), 28-38. https://doi.org/10.17561/jump.n3.4 


\section{Introducción}

Las relaciones de género han sido objeto de estudio durante las últimas décadas debido a las repercusiones que ejercen en el desarrollo integral de las personas (García-Bacete, 2006). Concretamente, el hecho de que en edades de preadolescencia aparezcan diferencias muy marcadas en las relaciones que se forjan entre los chicos y las chicas, puede tener importantes consecuencias posteriormente en su vida de preadolescentes (Garaigordobil y Aliri, 2013). García-Bacete et al. (2013) y Rebollo et al. (2017) han comprobado que, de manera generalizada, los niños desde la infancia y de manera más acusada durante la preadolescencia y adolescencia establecen sus relaciones sociales de manera intragénero. Esto tiene como consecuencia la limitación del aprendizaje de habilidades como la negociación o la resolución de conflictos, las cuales son fruto de las relaciones entre personas de distinto sexo (Navarro-Pertusa, 2004).

Si bien la creación de grupos que favorezcan las relaciones intergénero, parece una cuestión relevante para el desarrollo psicosocial de los niños, este tipo de relaciones no debe desarrollarse de cualquier manera. A tal fin, deben de tenerse en cuenta dos cuestiones, tales como la hegemonía masculina y los estereotipos de género. El concepto de hegemonía masculina se define como la configuración de la práctica de género que representa la respuesta aceptada actualmente al problema de la legitimidad del patriarcado, que se toma para asegurar la posición dominante de los hombres y la subordinación de la mujer (Connell, 2005). Tradicionalmente los agentes implicados en la práctica de deporte y actividad física han venido reforzando la versión dominante de la masculinidad (Frosh et al. 2002; Vidiella et al., 2010). Además, varios de los estudios que analizan la participación dentro del área de Educación Física (EF) muestran que, los niños suelen tener todavía un papel más protagonista en las clases y mostrar cierta superioridad sobre las chicas (Pope y O'Sullivan, 2003).

En cuanto a los estereotipos de género, Williams y Best (1990) definen estos como creencias de género compartidos socialmente, que traen consigo un impacto entre las relaciones de chicas y chicos. En EF, Soler (2009) mostró cómo en las clases también aparecen de manera inherente estas actitudes y comportamientos estereotipados. Esto es relevante ya que la interiorización de las diferencias de género y los estereotipos muestran repercusiones educativas en tanto que repercuten en la forma de pensar y actuar de los niños y en muchos de los casos los comportamientos y resultados que se esperan de ellos son fruto de estas concepciones.

Las repercusiones educativas fruto de estas concepciones estereotipadas transmitidas a lo largo de la historia afectan tanto a los chicos como a las chicas, pero no del mismo modo. Shen et al. (2012) señalaban como todavía se tiene una visión en la que las chicas deben ser dóciles, inactivas y preocupadas por la apariencia y los chicos, por el contrario, deben ser fuertes, agresivos y activos. En el término de las relaciones, García-Bacete et al. (2008) destacan que estas se siguen estableciendo de manera intragénero y son diferentes en función del género, pues las relaciones entre niños se caracterizan por estarformadas pormás miembros y están más preocupados por ostentar el puesto de líder. En cambio, las relaciones de las chicas se caracterizan por ser más intensas y formadas por menos miembros. Perry y Pauletti (2011) coinciden en que existen diferencias significativas en la configuración de estas relaciones, las cuales surgen antes en el tiempo en las chicas que en los chicos.

En EF se han utilizado diferentes estrategias para mejorar el clima social del aula y la mejora de la responsabilidad personal y social, tales como el modelo de Responsabilidad Personal y Social (Hellison, 1995) y el modelo de Aprendizaje Cooperativo (Fernández-Río y Méndez-Giménez, 2016). Además, otra de estas estrategias es el modelo pedagógico de Educación Deportiva (ED). La ED hace uso de dos pilares básicos para tratar de combatir este problema y desarrollar socialmente a los alumnos. El primer pilar es la existencia de seis elementos básicos (temporadas, competición, afiliación, festividad, registro de datos y evento culminante) que introducen a los alumnos en situaciones comunes de la vida diaria, que le obligarán a desenvolverse socialmente con sus compañeros. El segundo pilar hace referencia a la existencia de una serie de roles que los alumnos desarrollan (preparador físico, director deportivo, entrenador, árbitro...), y que obligarán al alumno a asumir una serie de responsabilidades en relación a sus compañeros. Así, la concepción que los niños tengan de la competición debe ser fruto de la superación individual y grupal que se consigue 
a través del trabajo en equipo y la cooperación (García-López y Gutiérrez, 2016), repercutiendo esto sobre las relaciones sociales de los alumnos.

En ED se han llevado a cabo diversos estudios referentes al género. Hastie (1998) desarrolló una experiencia durante 20 sesiones con 72 alumnos (37 niños y 35 niñas) con el objetivo examinar la participación y las percepciones de las niñas en un deporte colectivo, como el hockey. Así, comprobó la utilidad de la ED como medio para disminuir la marginación por motivos de género, aumentando la motivación, responsabilidad o relación y las oportunidades de participación durante situaciones de práctica y juego. Burgueño et al. (2019) analizaron el impacto de 12 sesiones de ED sobre la motivación de 75 alumnos (38 chicos y 37 chicas) de Bachillerato, considerando la influencia del género. De esta forma, comprobaron cómo evolucionan la motivación, responsabilidad o relación de manera distinta en los alumnos y alumnas a lo largo del desarrollo de las temporadas de ED. Así, observan que las chicas mejoran en términos de regulación introyectada, disminuyendo sus niveles de desmotivación y los chicos en cambio, lo hacen respecto a la regulación identificada. Del mismo modo, Puente-Maxera et al. (2020) en una experiencia con 96 estudiantes (53 chicos y 43 chicas) donde tenían como objetivo analizar los efectos de dos dinámicas de roles sobre los niveles de responsabilidad, encontraron que, la asunción de una responsabilidad y el establecimiento de normas permiten que se mejoren las relaciones entre iguales.

En todas estas intervenciones, la duración del programa es fundamental, de tal modo que Farias et al. (2017) indican que para que se obtengan cambios significativos en términos de igualdad y equidad no es suficiente con el desarrollo de una única temporada. Por otro lado, mucho tiene que ver la edad de los alumnos, tal y como se refleja en estudios como el de Chen y CurtnerSmith (2015), cuyos resultados muestran que las niñas de Educación Primaria pueden ser igual de competentes en términos físicos y de competitividad que los niños, diferencias que sí se observan a lo largo de la etapa de Educación Secundaria.

Si bien estudios anteriores se centraban en las variables de motivación o en el papel que desempeña cada género dentro del deporte, este estudio se focalizó en analizar las características de las relaciones existentes entre los chicos y chicas de una clase de sexto curso de Educación Primaria. Se prestó especial atención a la convivencia entre chicos y chicas, y a los motivos que determinan dichas relaciones, así como a la repercusión que sobre las mismas podía tener la implementación de una temporada de ED. En concreto, el objetivo del presente estudio fue abordar un caso en el que la situación inicial era de completo rechazo a la convivencia entre los chicos y las chicas, y de qué manera una temporada de ED favoreció las interacciones intergénero.

\section{Método}

Dada la naturaleza del estudio y los objetivos que se plantearon, se eligió un diseño cuasi experimental para el desarrollo. Este tipo de diseños experimentales se caracterizan por la elección de grupos naturales debido al acceso que se tiene a la muestra y por no incluir un grupo de control. Si bien estos diseños tienen limitada su validez externa, sin duda aportan una gran validez ecológica a sus resultados, pues permiten conocer en mayor profundidad el funcionamiento de los programas que estudian en su contexto real (Bryman, 2016).

\section{Participantes}

Los participantes fueron 23 alumnos ( 11 chicos y 12 chicas) con una media de edad $(\bar{X})$ de 11,7 años y una desviación estándar ( $\sigma$ ) de 0,62. Además, se contó con la participación de un docente de 60 años de edad y 37 años de experiencia docente. Su experiencia en el modelo consistía en un curso de formación de 20 horas, y la puesta en práctica con anterioridad de la misma temporada que se llevó a cabo en el estudio. El centro educativo en el que se llevó a cabo el presente estudio se trata de un centro público situado en una ciudad de tamaño medio del centro de España. Los alumnos pertenecían a una única clase de sexto curso de Educación Primaria y la muestra fue seleccionada por conveniencia. Según los datos ofrecidos por el centro educativo, las familias de los alumnos que han participado en el estudio pertenecen a una clase socioeconómica media.

\section{Programa de intervención}

El programa fue implementado en diez sesiones de 60 minutos cada una, repartidas en dos sesiones semanales. Del mismo modo, siguiendo las características básicas de la ED, en esta 
temporada se preservaron los aspectos básicos del deporte como son la afiliación, la competición, la temporada, el evento culminante, el registro de datos y la festividad (García-López y Gutiérrez, 2016). Las temporadas se dividieron en tres fases: pretemporada, temporada regular y fase final. La pretemporada estuvo centrada en el trabajo de los contenidos técnico-tácticos y reglamentarios, en la fase regular se desarrolló la competición y en fase final se disputaron las semifinales, la final y la fiesta de clausura. En la tabla 1 se puede consultar de manera esquemática el desarrollo de dicho programa.

La clase se dividió en cuatro equipos. Los equipos se formaron de manera equitativa teniendo en cuenta el nivel de habilidad de los alumnos a través de un juego inicial de selección, junto con una serie de medidas orientadas a la mejora de las relaciones intergénero. Por tanto, los propios alumnos configuraron los equipos siguiendo las directrices dictadas por el docente, según las cuales debían formas equipos mixtos. Además, los alumnos en consenso con su equipo fueron los encargados de distribuir los distintos roles para lo que también se estableció como requisito que todos los componentes estuviesen de acuerdo en dicha distribución. Así, se buscaba dotar a todos los componentes de voz y voto, intentando evitar que los chicos asignaran los roles por defecto a las chicas. En cuanto a la selección del deporte, se eligió el ringo porque se trata de un deporte alternativo donde tanto chicos como chicas parten de un nivel de juego similar. En este sentido, también se eligió por ser un deporte de cancha dividida, pues al ser el contacto físico menor, se evitan posibles enfrentamientos. Más adelante, en el desarrollo de las actividades complementarias se estableció el cambio de pareja obligatorio para favorecer las parejas mixtas.

Para asegurar la fidelidad al modelo, se concretaron reuniones semanales con el docente para solucionar las dudas que pudiera tener durante el desarrollo del programa y se realizó una visita en cada una de las fases de la temporada. Además, en estas reuniones se confirmó el correcto funcionamiento de los elementos básicos de la ED, los roles y los materiales curriculares empleados (hoja de selección de equipos, hoja de registro de responsabilidades de equipo y rol, actas de partidos, etc.). Para ello se siguieron una lista de comprobación desarrollada a partir de las de Metzler (2017) y Sinelnikov (2009).
Tabla 1. Desarrollo del programa de intervención

\begin{tabular}{|c|c|c|}
\hline Sesiones & Fase & Desarrollo \\
\hline Sesión 1 & Pretemporada & $\begin{array}{l}\text { Presentación de la metodología } \\
\text { y formación de equipos }\end{array}$ \\
\hline Sesión 2 & Pretemporada & $\begin{array}{l}\text { Trabajo del problema táctico: } \\
\text { mantener el móvil en juego }\end{array}$ \\
\hline Sesión 3 & Pretemporada & $\begin{array}{l}\text { Trabajo del problema táctico: } \\
\text { llevar la iniciativa en el juego }\end{array}$ \\
\hline Sesión 4 & Pretemporada & $\begin{array}{l}\text { Trabajo del problema táctico: } \\
\text { conseguir el objetivo }\end{array}$ \\
\hline Sesión 5 & Pretemporada & Partidos amistosos \\
\hline \multicolumn{3}{|c|}{ Actividad complementaria 1} \\
\hline Sesión 6 & Temporada & $\begin{array}{l}\text { Entrenamiento y partidos } \\
\text { oficiales }\end{array}$ \\
\hline Sesión 7 & Temporada & $\begin{array}{l}\text { Entrenamiento y partidos } \\
\text { oficiales }\end{array}$ \\
\hline Sesión 8 & Temporada & $\begin{array}{l}\text { Entrenamiento y partidos } \\
\text { oficiales }\end{array}$ \\
\hline \multicolumn{3}{|c|}{ Actividad complementaria 2} \\
\hline Sesión 9 & Fase final & Semifinales y gran final \\
\hline Sesión 10 & Fase final & $\begin{array}{l}\text { Evento culminante (entrega de } \\
\text { premios y diplomas) }\end{array}$ \\
\hline \multicolumn{3}{|c|}{ Actividad complementaria 3} \\
\hline
\end{tabular}

\section{Procedimiento}

El desarrollo del estudio se llevó a cabo en cuatro fases. En la primera fase, se realizaron una serie de entrevistas a los profesores que impartían clase a este grupo y en especial y más en profundidad al profesor de EF, para poder obtener más información sobre las relaciones entre los chicos y las chicas. Tras ello, en la segunda fase, los alumnos realizaron el primer test sociométrico que nos permitió conocer cómo estaban establecidas las relaciones entre ambos géneros.

Una vez recogida esta información, en la tercera fase se llevó a cabo la implementación del programa de intervención al cual se le dedicaron diez sesiones y se realizaron tres actividades complementarias. Por último, en la quinta fase los alumnos volvieron a completar el test sociométrico con el fin de comprobar si estas relaciones existentes habían mejorado tras la implementación del programa. Y, además, se realizó una entrevista final al docente de EF para conocer sus percepciones tras el desarrollo del programa.

\section{Instrumentos de medida}

En este programa se utilizaron tres instrumentos de medida, un test sociométrico y dos entrevistas al docente de EF. Estos instrumentos se utilizaron con el objetivo de conocer cómo estaban 
configuradas las relaciones entre ambos géneros en este grupo de alumnos. Del mismo modo, para tener en cuenta la evolución de dichas relaciones a lo largo de la implementación de la temporada de ED, se realizó un análisis posterior tras la aplicación de dicho modelo, comparando los resultados con la situación inicial.

El test sociométrico (Moreno, 1972), el cual se suministró tanto antes como después del programa, muestra una fiabilidad, a test-retest = .72 (García-López et al., 2012). Dicho instrumento permitió conocerla configuración delas relaciones entre los alumnos y su evolución tras el programa de intervención. Este estaba formado por cuatro preguntas a las cuáles los alumnos podían dar un máximo de tres respuestas, no pudiendo repetir ningún nombre dentro de la misma pregunta y donde debían escribir el motivo de elegir a cada compañero o compañera. Los motivos fueron categorizados siguiendo el estudio de Monjas et al. (2008) y las categorías seleccionadas fueron: comportamiento, amistad, compañerismo, dominancia, falta de competencia y otros. Las preguntas incluidas y fueron las siguientes:

- ¿Con qué tres compañeros/as te gustaría hacer los ejercicios de clase o estudiar? (Compañero positivo).

- ¿Con qué tres compañeros/as no te gustaría formar un grupo para hacer los ejercicios de clase o estudiar? (Compañero negativo).

- ¿Con qué tres compañeros/as te gustaría jugar en tu tiempo libre? (Amigo positivo).

- ¿Con qué tres compañeros/as no te gustaría jugar en tu tiempo libre? (Amigo negativo).

En segundo lugar, se realizaron dos entrevistas semiestructuradas (Cohen y Manion, 1990) al docente de EF con el objetivo de recabar toda la información necesaria sobre su percepción de la situación. Las entrevistas fueron diseñadas ad hoc y revisadas por expertos con más de diez años de investigación en ED, con el fin de asegurar la confiabilidad del proceso. En estas entrevistas se realizaron preguntas tales como: ¿has observado algún distanciamiento entre los chicos y las chicas en tus clases? ¿Es algo que observaste al principio de curso o que ha ido apareciendo progresivamente? ¿Crees que esta separación entre ambos géneros es algo propio de la edad o particular de este grupo? Tras el desarrollo del programa se efectuó otra entrevista al especialista de EF donde se incluyeron preguntas como las que a continuación se incluyen: tras la implementación de la temporada y las actividades complementarias, ¿dirías que ha mejorado la relación entre ambos géneros? ¿Por qué?; ¿Cómo definirías la situación inicial del grupo y la situación final?; ¿Qué puntos fuertes crees que tiene la ED para abordar este tipo de problemática?

\section{Análisis de los datos}

Para llevar a cabo el análisis cuantitativo de los resultados sehizo uso del programa estadístico IBM SPSS Statistics 24. Se calcularon los estadísticos descriptivos y se aplicó la prueba t de student para muestras relacionadas con lafinalidad de comparar las respuestas de aceptación y rechazo obtenidas en el pre-test y post-test y comprobar si existían cambios significativos entre ambas. Se aportan los valores de $t$ resultantes de dichas pruebas en función de los grados de libertad, así como las diferencias se consideraron estadísticamente significativas para valores $p<0.05$. El tamaño del efecto se calculó y fue interpretado usando los rangos recomendados por Hopkins et al. (2009): efecto bajo $(0,1)$, moderado $(0,3)$, alto $(0,5)$, muy alto $(0,7)$ y extremadamente alto $(0,9)$. Un tamaño del efecto moderado hace referencia a la magnitud alcanzada con el programa sobre esta muestra seleccionada. Las entrevistas realizadas para conocer las percepciones del docente de EF fueron grabadas en audio y transcritas literalmente. A continuación, se aplicó un análisis de datos convencional (Hsieh y Shannon, 2005; Tesch, 1990), según el cual primero se realizó una lectura de las entrevistas para obtener una perspectiva global de los datos obtenidos, después se hizo una segunda lectura en la que se tomaron notas y se derivaron los códigos, que finalmente se agruparon en categorías. A partir de estas categorías se realizó una descripción narrativa (Bryman, 2016). Para el análisis de la entrevista se utilizó el programa informático ATLAS.ti versión cloud.

\section{Resultados}

A continuación, se presentan los resultados que se desprenden del sociograma realizado a los alumnos y las entrevistas realizadas al profesor. 


\section{Respuestas de aceptación y rechazo entre los alumnos de ambos géneros}

Del análisis de los datos del sociograma en el pre-test se desprende un rechazo evidente entre chicos y chicas en ambos sentidos, si bien en el caso de los chicos no es total como sí lo es en el caso de las chicas. En cuanto a lo que aceptaciones se refiere, se puede destacar que, en el caso del compañero positivo, el $0 \%$ de las chicas es aceptado por los chicos y que, en cambio, el $6 \%$ de los chicos es aceptado por las chicas. Se obtuvieron los mismos resultados en el amigo positivo. Respecto a los rechazos, en la variable de compañero negativo, el $87 \%$ de las chicas es rechazado por los chicos y el $77 \%$ de los chicos es rechazado por las chicas. En relación al amigo negativo, el 93\% de las chicas es rechazado por los chicos y el $78 \%$ de los chicos es rechazado por las chicas.

En cuanto a los motivos de aceptación y rechazo dados, las respuestas fueron categorizadas en función del género y de las categorías descritas por Monjas et al. (2008): comportamiento, amistad, compañerismo, dominancia, falta de competencia y otros. En cuanto a los motivos de aceptación reflejados en este test, los chicos destacan la categoría de amistad y en el caso de los rechazos, destacan la categoría de comportamiento. Por otro lado, en cuanto a las chicas, destaca la categoría de comportamiento en ambos casos.

Tras la implementación del programa, los resultados con respecto a las aceptaciones sufrieronunligero cambio. Enel caso delcompañero positivo, el $6 \%$ de las chicas es aceptado por los chicos y que el $9 \%$ de los chicos es aceptado por las chicas. Así, en el amigo positivo se obtuvo el mismo porcentaje de aceptación de los chicos hacia las chicas. En cambio, la aceptación de las chicas hacia los chicos se incrementa hasta un $14 \%$. En cuanto a rechazos, las respuestas reflejan que el $83 \%$ de las chicas es rechazado por los chicos y que el $74 \%$ de los chicos es rechazado por las chicas. En la variable de amigo negativo, el 81\% de las chicas es rechazado por los chicos y el $71 \%$ de los chicos es rechazado por las chicas.

En comparación con el pre-test, se reafirman los motivos que chicos y chicas enuncian para justificar sus aceptaciones y rechazos. En las aceptaciones por parte de los chicos, la categoría más destacada sigue siendo la de amistad y en el caso de los rechazos, la categoría con mayor número de respuestas sigue siendo la de comportamiento. En el caso de las chicas, como sucedía en el pre-test destacan las categorías de comportamiento.

Las comparaciones entre la situación inicial y final en cada una de las variables se pueden consultar de manera sencilla en la tabla 2.

Por todo ello, los resultados de este instrumento muestran un claro rechazo inicial entre ambos géneros, siendo las aceptaciones por parte de los chicos hacia las chicas nulas. Tras el programa, se constatan mejoras en la aceptación por parte de ambos géneros, estas se incrementan un $6 \%$ en el caso de los chicos hacia las chicas en ambas variables (compañero y amigo positivo) y un $3 \%$ en el caso de las chicas hacia los chicos en el compañero positivo y un $8 \%$ en el caso del amigo positivo.

Tabla 2. Evolución de los porcentajes en cada una de las variables con respecto al género contrario

\begin{tabular}{lllll}
\hline & \multicolumn{2}{c}{ Chicos } & \multicolumn{2}{c}{ Chicas } \\
\cline { 2 - 5 } & Pre-test & Post-test & Pre-test & Post-test \\
\hline $\begin{array}{l}\text { Compañero } \\
\text { positivo }\end{array}$ & $0 \%$ & $6 \%$ & $6 \%$ & $9 \%$ \\
$\begin{array}{l}\text { Amigo } \\
\text { positivo }\end{array}$ & $0 \%$ & $6 \%$ & $6 \%$ & $14 \%$ \\
$\begin{array}{l}\text { Compañero } \\
\text { negativo }\end{array}$ & $87 \%$ & $83 \%$ & $77 \%$ & $74 \%$ \\
$\begin{array}{l}\text { Amigo } \\
\text { negativo }\end{array}$ & $93 \%$ & $81 \%$ & $78 \%$ & $71 \%$ \\
\hline
\end{tabular}

Además, estos también constatan una disminución del rechazo hacia el género contrario tanto en el caso de los chicos como de las chicas. En el caso de los chicos hacia las chicas, en el compañero negativo este disminuye un $4 \%$ y en el amigo negativo un $12 \%$. En el caso de las chicas hacia los chicos, este disminuye en el caso del compañero negativo un $3 \%$ y en el de amigo negativo un $7 \%$. Dicho esto, estas diferencias solo fueron significativas en el caso de la variable amigo positivo, con un valor de $t(22)=-2,472, p=.22$, la cual obtuvo un valor del tamaño del efecto moderado $(\mathrm{d}=$ ,422). La variable de compañero positivo muestra un tamaño del efecto que se puede considerar también moderado $(\mathrm{d}=344)$, y las variables de compañero y amigo negativo un tamaño de efecto alto ( $d=, 548 ; \mathrm{y} d=, 518$, respectivamente) (tabla 3$)$. 
Tabla 3. Diferencias en las aceptaciones y rechazos a compañeros del género contrario entre la situación inicial y final

\begin{tabular}{lcccl}
\hline \multicolumn{5}{c}{ Prueba t para muestras relacionadas } \\
& t & gl & $\begin{array}{c}\text { Sig. } \\
\text { (bilateral) }\end{array}$ & d de Cohen \\
\hline Compañero positivo & $-1,817$ & 22 &, 083 &, 344 \\
Amigo positivo & $-2,472$ & 22 &, 022 &, 422 \\
Compañero negativo & 1,141 & 22 &, 266 &, 548 \\
Amigo negativo & 2,011 & 22 &, 057 &, 518 \\
\hline
\end{tabular}

\section{Percepción del docente}

Con respecto al primer objetivo que se planteó, estudiar las relaciones sociales entre ambos géneros, para obtener información se realizaron dos entrevistas al especialista de $\mathrm{EF}$, una antes del programa y otra al término del mismo. Por ello, a continuación, se detallan los resultados obtenidos en dichas entrevistas.

En dichos resultados, se comienza destacando la razón que provoca la realización del presente trabajo, el rechazo entre ambos géneros. Así, la percepción de esta situación viene determinada por una serie de motivos que aporta el propio docente para los cuales hace referencia tanto a su práctica docente como a la metodología empleada. Por último, se recogen los frutos de la implementación del programa en forma de beneficios y limitaciones.

Manuel (anonimizado), el maestro de EF que imparte clase a este grupo, describió que la situación inicial entre los chicos y las chicas era de claro rechazo, basado en la estable intención desde ambos lados de no mezclarse: "Los chicos se sientan a un lado juntos, dejan un espacio y en el otro lado se sientan todas las chicas juntas" (Entrevista 1, E1). Esto es especialmente problemático a la hora de hacer equipos mixtos, ya que "no quieren jugar juntos en los mismos equipos" (Manuel, E1).

A la hora de identificar los motivos que provocan este claro rechazo, Manuel fue incapaz de identificar una razón evidente causante de la situación, pero sí afirmó de forma segura que no era una situación provocada únicamente por la edad: "Yo creo que no es algo propio de la edad porque como te decía antes doy clase al resto de cursos de sexto y esta circunstancia no aparece de manera tan marcada como en esta clase" (E1).

En cualquier caso, Manuel describe como esta situación está condicionando el desarrollo normal de sus clases:
Manuel: "Esta circunstancia sí que me ha condicionado porque con mi forma de dar las clases digamos que, usando una metodología tradicional, se originaban bastantes conflictos. A la hora de hacer yo los equipos para los juegos nunca estaban de acuerdo, pues yo no voy a jugar con tal porque no da una, con este no vamos a tocar el balón, no corre..." (E1).

En relación a la ED, Manuel afirmó el carácter novedoso de esta en su práctica profesional. A pesar de la escasa experiencia y dominio del modelo reconoció claramente su carácter innovador dentro de las clases de EF y el grado de implicación que despierta en los alumnos, consiguiendo que estos se involucren de manera activa en sus aprendizajes y mejoren notablemente su responsabilidad.

Manuel: "Creo que esta metodología tiene muchas cosas buenas, implica mucho a los alumnos y adquieren aprendizajes siendo ellos los protagonistas. No es perfecta pero el hecho de jugar por equipos y tener responsabilidades creo que saca lo mejor de cada alumno porque no quieren perjudicar al resto" (Entrevista 2, E2).

Además, Manuel destacó especialmente la motivación e implicación de los alumnos en el trabajo en equipo: "la pertenencia a un equipo une mucho a esos alumnos que quieren al fin y al cabo conseguir un objetivo común". Y en ello veía la razón por la que los conflictos se reducen a lo largo de la temporada: "sin duda se han reducido los conflictos y chicos y chicas han compartido equipos jugando muy bien, ejerciendo sus roles y respetando las normas de deportividad" (E2).

Tras ello, Manuel identificaba algunos de los motivos que le conducían a pensar que tras el programa se habían reducido los rechazos y se había conseguido un mayor acercamiento entre ambos géneros:

"He visto cómo se buscaban unos a otros para hacer las actividades, muchas veces se buscaban porque sabían que ha determinado chico o chica se les da bien eso, pero oye, aunque sea un poco por conveniencia es un paso para comenzar a acercarse" (E2).

En este sentido, él mismo destacó que estas mejoras se aprecian más allá de las clases y lo ha 
podido observar en otros momentos y situaciones, como son los cambios de clase: "cuando vamos de camino al gimnasio o al patio veo a chicos y chicas hablar o dentro de los propios equipos veo que hay comunicación entre todos, hablan, acuerdan cosas, piensan estrategias" (E2).

Para concluir, Manuel señalaba las limitaciones que encontró a lo largo de la experiencia, destacando entre ellas la escasez de tiempo para llevar a cabo el programa y el carácter aislado de la misma:

Manuel: "Hemos tenido poco tiempo por desgracia y es difícil que solo con esta experiencia consigamos que de la noche a la mañana sean súper amigos, pero por lo menos hemos puesto la primera pieza del puzzle" (E2).

\section{Discusión}

El objetivo que se perseguía con el presente estudio fue estudiar las relaciones sociales entre ambos géneros de una clase de sexto de Primaria, observando su evolución antes y después de la implementación de una temporada de ED. Tras el desarrollo del programa de intervención, se puede destacar la clara separación inicial entre ambos géneros y las mejoras obtenidas con el aumento de aceptaciones y la disminución de rechazos. En este sentido, la situación de partida coincide con lo que ya García-Bacete (2006) afirmaba, pues esta situación no es algo extraño ya que se constata a lo largo de toda la infancia y es que los niños y niñas prefieren a aquellos que tienen características similares a las suyas.

Al término del programa, el hecho de que se haya mejorado más la variable amigo positivo que compañero positivo, nos indica que los alumnos muestran una mayor disposición para relacionarse con sus compañeros de distinto género sobre todo en contextos lúdicos. El hecho de que tras el programa se hayan incrementado las aceptaciones y reducido los rechazos, permite considerar la afiliación a un equipo, elemento básico de la ED (Siedentop, 1994), un factor clave en la consecución de estos resultados. Los alumnos forman parte de un equipo estable lo que contribuye a que se establezcan vínculos y relaciones más estrechas entre ellos, circunstancia que en otras condiciones no se daría. En definitiva, la necesidad de que exista comunicación dentro del equipo para lograr los objetivos comunes permite que estos aprendan a regular sus conductas dentro del aula. MacPhail et al. (2004) en un estudio con niños de entre nueve y diez años destacaron un notable cambio en la disposición de los alumnos para relacionarse con compañeros que no formaban parte de su círculo de amigos gracias a la pertenencia a un mismo equipo. Estos resultados coinciden con nuestro estudio. Los alumnos reconocen que gracias a la pertenencia a un mismo equipo han tenido la oportunidad de entablar relaciones con compañeros con los que nunca habían tenido un contacto cercano y esto sin duda se ha visto reflejado en la disminución de los rechazos y los conflictos que se originaban a lo largo de las clases.

De estos resultados se desprende que los rechazos disminuyen más que suben los aciertos. Sin duda una disminución de los rechazos es un paso importante de cara a la posible consecución posterior de un aumento de aceptaciones. Además, es interesante comentar el hecho de que no se alcancen las 69 respuestas, pues ello supone que hay chicos y chicas que ya no llegan a rechazar a tres o más compañeros del otro género. En consecuencia, estos cambios implican que hay una tendencia hacia la aceptación entre los participantes de ambos géneros. Es más, parece natural que esa aceptación no sea necesario que se dé de cara al disfrute del tiempo libre, pero sí de cara a una colaboración durante su trabajo en clase.

Por tanto, como se menciona anteriormente, la afiliación a un equipo repercute en los comportamientos sociales de los alumnos y puede hacerlo con diferentes matices. Luna et al. (2020) enfatizan la idea de que los resultados obtenidos con adolescentes a través de la ED pueden estar más cerca de obtener la eficacia en la interacción social que a la aceptación dentro de un grupo. En cambio, en el presente estudio los resultados muestran como las interacciones surgidas dentro de un grupo concreto permiten la mejora de las relaciones a un nivel más general e incluso se pueden extrapolar fuera del aula. Como ya apuntaban Kirk y Kinchin (2003), la ED permite la transferencia de los conocimientos a otros ámbitos fuera del aula, hecho que el docente ha comenzado a observar en este grupo de alumnos a raíz del desarrollo de nuestro programa y es que no solo se adquieren conocimientos deportivos 
sino también sociales, como la empatía. GarcíaLópez y Gutiérrez (2015) encontraron mejoras en la empatía de alumnos de entre 11 y 14 años tras un programa de ED y es que los alumnos a través del desempeño de roles aprenden a ponerse en la piel de los demás, lo que permite a su vez, reducir los conflictos que surgen entre ellos.

En cuanto a la percepción del docente, esta reflejaba una clara situación de rechazo entre los alumnos de ambos géneros, vivenciada de forma habitual en sus clases. Sin embargo, aunque concebía la edad como una de las razones que motivaban esta separación, y sabía que no era la única, era incapaz de identificar el resto de los motivos. En cambio, los alumnos perciben con claridad el motivo principal que dan ambos géneros para rechazar a sus compañeros de distinto género, y este es el comportamiento. Los chicos consideran que las chicas quieren llevar siempre las riendas en las decisiones y, por el contrario, las chicas que los chicos se distraen fácilmente y son muy desordenados. Estas respuestas coinciden con los resultados obtenidos por García-Bacete et al. (2010), que indican que el comportamiento es una de las razones importantes de rechazo entre niños y niñas y, sobre todo, aquellos que reflejan falta de atención o resultan molestos. Ante esta realidad concreta, la temporada de ED propició un entorno seguro de aprendizaje (Farias et al., 2017) que permitió a los alumnos disfrutar de la experiencia cambiando su forma de concebir las cosas. La seguridad en el ambiente de aprendizaje, entendida como la posibilidad de relacionarse con los otros sin correr riesgos emocionales, es algo que también se ha conseguido en estudios previos tales como los de Ennis et al. (1999) y Farias et al. (2017).

Con respecto a las limitaciones encontradas, se destaca el período de tiempo limitado del que se dispuso para poner en práctica el programa. Debido a esto, se desarrollaron un número de sesiones inferior al recomendado por Siedentop et al. (2011), pues estos aconsejan superar las 15 sesiones de duración. El hecho de que los alumnos se encontrasen en la recta final de su paso por la etapa de Educación Primaria y el programa se desarrollase en el último trimestre escolar, limitó la duración de este. Además, otra de las limitaciones encontradas fue la dificultad de acceso a un mayor número de participantes y docentes, por lo que finalmente el tamaño de la muestra quedó reducido a un único grupo de alumnos y un docente especialista de EF.

Por todo esto, poniendo la vista en investigaciones futuras, sugerimos el comprobar la eficacia del modelo ED en la mejora en las relaciones sociales entre ambos géneros en situaciones devulnerabilidad social y discapacidad.

\section{Conclusiones}

Como en otras ocasiones, el modelo de ED se ha mostrado eficaz en el tratamiento de las cuestiones de género dentro del área de EF. En este caso concreto, se han mejorado las relaciones entre ambos géneros a través de la disminución de los rechazos y el aumento de las aceptaciones. De esta manera, este modelo pedagógico puede considerarse como un recurso que ofrece en el contexto educativo muchos beneficios más allá de los estrictamente deportivos. Un programa que incorpora medidas específicas junto con el buen hacer del docente fomenta el desarrollo de habilidades y comportamientos sociales que repercuten de forma positiva en la convivencia entre ambos géneros. Los comportamientos sociales, los cuales son enunciados por los alumnos como el principal motivo de rechazo, resultan así claves en el desarrollo de relaciones sociales positivas entre chicos y chicas. Por ello, desde nuestro ámbito se debe trabajar para que a través del desarrollo de habilidades sociales se mejoren las relaciones entre el alumnado de ambos géneros y se eliminen las concepciones estereotipadas transmitidas de forma tradicional en el contexto deportivo.

\section{Aplicaciones Prácticas}

En relación con los fines planteados en este trabajo se puede destacar la importancia de emprender medidas para fomentar dentro del aula las interacciones intergénero que en este rango de edades comienzan a tambalearse. De esta forma, para ello es necesario valorar la utilización de medidas o programas que, aunque estén centrados en la enseñanza de un contenido deportivo incluyan específicamente medidas que permitan mejorar las relaciones entre ambos géneros. Actualmente, son diversos los modelos que hacen uso de diferentes estrategias para mejorar el clima 
social del aula y la responsabilidad personal y social del alumnado, tales como el Aprendizaje Cooperativo (Fernández-Río y MéndezGiménez, 2016) oel modelo de Responsabilidad Personal y Social (Hellison, 1995), pero en este trabajo se muestran los resultados obtenidos por la ED, otra metodología que ha constatado obtener grandes resultados en la mejora de las relaciones sociales del alumnado (GarcíaLópez y Gutiérrez, 2016).

Los elementos que componen esta metodología, la competición, la afiliación o la asunción de roles permiten al alumnado adquirir aprendizajes que más tarde podrán extrapolar a su experiencia deportiva fuera de contexto escolar. De ahí la importancia de usar este modelo para eliminar actitudes estereotipadas y mejorar las interacciones intergénero, ya que los aprendizajes adquiridos en esta área podrán extenderse a otros contextos de su vida diaria. Además, el hecho de que los alumnos pertenezcan durante una temporada a un mismo equipo les ayuda a desarrollar ese sentimiento de equipo, el cual permite mejorar la relación entre los miembros y despertar en ellos un vínculo de lucha por un objetivo común. Por consiguiente, que los alumnos desempeñen sus propios roles favorece no solo la participación activa del alumnado en su proceso de enseñanzaaprendizaje sino también la asunción de responsabilidades a las cuales van ligadas valores de respeto o empatía, entre otras. Los alumnos aprenden a ponerse en la piel de sus compañeros, comprendiendo así la dificultad de tomar decisiones que repercuten a todos y comprendiendo los sentimientos derivados del desempeño de determinadas actitudes.

En este sentido, para que los beneficios que ofrece la metodología se puedan materializar es imprescindible el desarrollo de programas de una duración adecuada (diez-15 sesiones) y los cuáles se desarrollen de forma continuada en el tiempo. Esta experiencia aislada refleja la consecución de resultados positivos, pero para obtener cambios realmente significativos es necesario llevar a cabo un trabajo consciente y continuado que no se consigue con el desarrollo de una sola temporada. Por ello, es fundamental que este tipo de experiencias además de tener la duración adecuada incluyan medidas más específicas para conseguir el fomento de las relaciones intergénero (establecer normas en la formación de los equipos, favorecer los acercamientos, elegir un deporte novedoso que no suponga por defecto la superioridad de chicos o chicas...). Por lo tanto, la combinación de este de tipo de iniciativas junto con el uso de esta metodología y el buen hacer del docente, podrán asegurar el desarrollo de experiencias muy enriquecedoras tanto para alumnos como docentes y por supuesto, la mejora de la competencia social del alumnado.

\section{Referencias}

Bryman, A. (2016). Social Research Methods. Oxford University Press.

Burgueño, R., Martín, B., Morales, E., \& Medina-Casaubón, J. (2019). Influence of sport education on high school students' motivational response: A gender perspective. Retos. Nuevas tendencias en Educación Física, Deporte y Recreación, 37(37), 546-555. https://doi.org/10.47197/retos.v37i37.70880

Chen, Y., \& Curtner-Smith, M. D. (2015). Hegemonic masculinity in sport education: case studies of pre-service physical education teachers with teaching orientations. Sport, Education \& Society, 20(4), 546-563. https://doi.org/10.1080/13573322.2013.7751 16

Cohen, L., \& Manion, L. (1990). La entrevista. En Cohen, L. \& Manion, L. Métodos de investigación educativa. La Muralla.

Connell, R.W., \& Messerschmidt., J. W. (2005). Hegemonic masculinity: Rethinking the concept. Gender \& Society 19(6), 829-859. https://doi.org/10.1177/0891243205278639

Ennis, C. D., Solmon, M. A., Satina, B., Loftus, S. J., Mensch, J., \& McCauley, M. T. (1999). Creating a Sense of Family in Urban Schools Using "Sport for Peace" Curriculum. Research Quarterly for Exercise \& Sport, 70(3), 273-285. https://doi.org/1 0.1080/02701367.1999.10608046

Farias, C., Hastie, P. A., \& Mesquita, I. (2017). Towards a more equitable and inclusive learning environment in sport education: Results of an action research-based intervention. Sport, Education and Society, 22(4), 460-476. https://doi.org/10.108 0/13573322.2015.1040752

Fernández-Río, J., \& Méndez-Giménez, A., (2016). El Aprendizaje Cooperativo: Modelo Pedagógico para Educación Física. Retos. Nuevas tendencias en Educación Física, Deporte y Recreación, 29, 201-206. https://doi.org/10.47197/retos.v0i29.38721

Frosh, S., Phoenix, A., \& Pattman R. (2002). Young Masculinities: Understanding Boys in Contemporary Society. Palgrave.

Garaigordobil, M., y Aliri, J. (2013). Relaciones del sexismo con justificación de la violencia, y con otras formas de prejuicio como la dominancia social y el autoritarismo. Estudios de Psicología, 34(2), 127-139. https://doi. org/10.1174/021093913806751384

García- Bacete, F. J., Sureda, I. y Monjas, M. I. (2010). El rechazo entre iguales en la educación primaria: Una panorámica general. Anales de psicología, 26(1), 123-136.

García-Bacete, F. J. (2006). La identificación de los alumnos rechazados: Comparación de métodos sociométricos de nominaciones bidimensionales. Infancia y Aprendizaje, 29(4), 437-451. https://doi.org/10.1174/021037006778849585

García-Bacete, F. J., Sureda, I., y Monjas, M. I. (2008). Distribución sociométrica en las aulas de chicos y chicas a lo largo de la escolaridad. Revista de Psicología Social, 23(1), 63-74. 
García-Bacete, F. J., Rubio-Barreda, A., Milan-Rojas, I., y MarandePerrín G. (2013). El aprendizaje de la amistad en la Educación Primaria. Un procedimiento intensivo para ayudar a los niños rechazados a hacer amigos. Apuntes de Psicología, 31, 155163.

García-López, L. M., \& Gutiérrez, D. (2015). The effects of a sport education season on empathy and assertiveness. Physical Education and Sport Pedagogy, 20(1), 1-16. https://doi.org/10. 1080/17408989.2013.780592

García-López, L. M., Gutiérrez, D., González-Vílora, S., \& ValeroValenzuela, A. (2012). Cambios en la empatía, la asertividad y las relaciones sociales por la aplicación del modelo de instrucción educativa deportiva. Revista de Psicología del Deporte, 21(2), 321-330.

García-López, L.M., y Gutiérrez, D. (2016). Aprendiendo a Enseñar Deporte. Modelos de Enseñanza comprensiva y Educación deportiva. INDE.

Hastie, P. A. (1998). The Participation and Perceptions of Girls Within a Unit of Sport Education. Journal of Teaching in Physical Education, 17(2), 157-171.

Hellison, D. (1995). Teaching responsibility through Physical Activity. Champaing: Human Kinetics.

Hopkins, W., Marshall, S., Batterham, A., \& Hanin, J. (2009). Progressive statistics for studies in sports medicine and exercise science. Medicine Science in Sports Exercise, 41(1), 3. https://doi.org/10.1249/MSS.0b013e31818cb278

Hsieh, H. F., \& Shannon, S. E. (2005). Three Approaches to Qualitative Content Analysis. Qualitative Health Research, 15(9), 12771288. https://doi.org/10.1177/1049732305276687

Kirk, D., \& Kinchin, G. (2003). Situated learning as a theoretical framework for sport education. European physical education review, 9(3), 221-235.

Luna, P., Guerrero, J., Rodrigo-Ruiz, D., Losada, L., \& Cejudo, J. (2020). Social Competence and Peer Social Acceptance: Evaluating Effects of an Educational Intervention in Adolescents. Frontiers in Psychology, 11, 1305. https://doi. org/10.3389/fpsyg.2020.01305

MacPhail, A., Kirk, D., \& Kinchin, G. (2004). Sport education: Promoting team affiliation through physical education. Journal of teaching in Physical Education, 23(2), 106-122.

Metzler, M. W. (2017). Instructional models for physical education (3 ed.). Routledge

Monjas, M. I., Sureda, I., \& García-Bacete, F. J. (2008). ¿Por qué los niños y las niñas se aceptan y se rechazan? Cultura y Educación, 20(4), 479-492. https://doi. org/10.1174/113564008786542181

Moreno, J. L. (1972). Fundamentos de la sociometría. Paidós.

Navarro-Pertusa, E. (2004). Género y relaciones personales íntimas. En E. Barberá-Heredia y I. Martínez-Benlloch (Coords.), Psicología y Género (pp. 171-192). Pearson Educación.

Perry, D. G., \& Pauletti, R. E. (2011). Gender and Adolescent Development. Journal of Research on Adolescence, 21(1), 6174. https://doi.org/10.1111/j.1532-7795.2010.00715.x

Pope, C., \& O'Sullivan, M. (2003). Darwinism in the gym. Journal of Teaching in Physical Education, 22, 311-327. https://doi. org/10.1123/jtpe.22.3.311

Puente-Maxera, F., Mahedero, P., Méndez-Giménez, A., \& Martínez de Ojeda Pérez, D. (2020). Educación Deportiva, roles y vulnerabilidad. Influencia en la responsabilidad y la competencia interculturalidad en adolescentes (78).

Rebollo, Á., Ruiz, E., \& García, R. (2017). Preferencias relacionales en la adolescencia según el género. Revista Electrónica de Investigación Educativa, 19(1), 58-72. https://doi. org/10.24320/redie.2017.19.1.1022

Shen, B., McCaughtry, N., Martin, J., Fahlman, M., \& Gran, A. (2012). Urban High-School Girls' Sense of Relatedness and Their Engagement in Physical Education. Journal of Teaching in Physical Education, 31, 231-245.

Siedentop, D. (1994). Sport education: Quality PE through positive sport experiences. Human Kinetics.
Siedentop, D., Hastie, P. A., \& Van der Mars, H. (2011). Complete Guide to Sport Education ( $2^{\mathrm{a} e d}$ ). Human Kinetics.

Sinelnikov, O. A. (2009). Sport education for teachers: Professional development when introducing a novel curriculum model. European Physical Education Review, 15, 91-114.

Soler, S. (2009). Los procesos de reproducción, resistencia y cambio de las relaciones tradicionales de género en Educación Física: el caso del fútbol. Cultura y Educación, 21(1), 31-42. https://doi.org/10.1174/113564009787531253

Tesch, R. (1990). Qualitative Research Analysis Types and Software Tools. Falmer Press.

Vidiella, J., Herraiz, F., Hernández, F., y Sancho, J. M. (2010). Masculinidad hegemónica, deporte y actividad física. Movimento, 16(4), 93-115. https://doi.org/10.22456/19828918.15031

Williams, J.E., \& Best, D.L. (1990). Measuring Sex Stereotypes: A Multination Study. Sage. 